\title{
Suppression of p21 by c-Myc through members of miR-17 family at the post-transcriptional level
}

\author{
ZAOZAO WANG, MEI LIU, HONGXIA ZHU, WEI ZHANG, SHUN HE, \\ CHENFEI HU, LANPING QUAN, JINFENG BAI and NINGZHI XU
}

\begin{abstract}
Laboratory of Cell and Molecular Biology \& State Key Laboratory of Molecular Oncology, Cancer Institute \& Cancer Hospital, Chinese Academy of Medical Sciences \& Peking Union Medical College, Beijing 100021, P.R. China
\end{abstract}

\author{
Received June 1, 2010; Accepted July 26, 2010
}

\section{DOI: 10.3892/ijo_00000783}

\begin{abstract}
The miR-17 family, composed of miR-17-5p, miR-20a/b, miR-106a/b and miR-93, has been demonstrated to take part in critical pathways that regulate cellular life and death decisions during normal development and in malignancy. Previous studies have shown that the expression of miR-17 family members has close relationship with the expression of c-Myc. Another study has reported p21 is a direct target of miR-17 family and their silencing of p21 contributes to tumor cell proliferation in part. Since c-Myc is a potent transcriptional repressor of $\mathrm{p} 21$, these findings suggest that c-Myc may negatively modulate p21 expression through an additional pathway except transcriptional suppression. In the study presented here, we compared p21 mRNA in the nucleus and cytoplasm of c-Myc stable transfectants with its control, which indicated further repression of p21 by c-Myc at the post-transcriptional level. Stem-loop and conventional real-time PCR showed elevated expression of some members in miR-17 family and their primary transcripts when c-Myc was stably overexpressed. To further investigate the relationship of c-Myc, miR-17 family and p21, we antagonized the expression of each miR-17 family member by transfection of their antisense oligonucleotides respectively in transfectants constitutively overexpressing c-Myc or not, and found that the restoration of p21 expression by treatment above was much stronger in the presence of c-Myc. These results suggest c-Myc could further repress p21 expression at the posttranscriptional level through some, but not all, members of miR-17 family.
\end{abstract}

\section{Introduction}

p2 $1^{\text {Cip1/Wafl }}$ (hereafter referred to as p21), the founding member of the Cip/Kip family of cyclin-dependent kinase (CDK)

Correspondence to: Dr Ning-Zhi Xu, Laboratory of Cell and Molecular Biology \& State Key Laboratory of Molecular Oncology, Cancer Institute \& Cancer Hospital, Chinese Academy of Medical Sciences \& Peking Union Medical College, Panjiayuan, Chaoyang District, P.O. Box 2258, 100021, Beijing, P.R. China

E-mail: xningzhi@public.bta.net.cn

Key words: c-Myc, p21, miR-17 family inhibitors, negatively regulates cell cycle progression by inhibiting a broad range of cyclin/Cdk complexes (1). Being a central player in cell cycle regulation, p21 is regulated by a variety of mechanisms. Among them, down-modulation of p21 level by c-Myc has been found to be important in a wide variety of circumstances (2-7). Many studies have been undertaken to decipher the mechanistic basis of p21 repression by c-Myc. Some suggested that c-Myc may bind to and inhibit the activity of the transcription factor Miz-1, thereby preventing Miz-1 from activating transcription of p21 (6). While others indicated that c-Myc may also interact with and antagonize the activity of additional transcriptional regulators, such as $\mathrm{Sp} 1 / \mathrm{Sp} 3$ (3), thus suppress the transcription of $\mathrm{p} 21$. Whereas the exact mechanism is still not fully understood.

Recently, many studies have identified that miRNAs in miR-17 family (also called miR-106b family) could regulate cell cycle progression through targeting p21 (8), and overexpression of miR-17 family members would contribute to tumorigenesis partly due to their deregulation of p21 (8-10). miR-17 family is composed of miR-17-5p, miR-20a (mature miRNAs arisen from miR-17-92 cluster located at 13q31.3), miR-106b, miR-93 (from miR-106b-25 cluster at 7q22), miR-106a and miR-20b (from miR-106a-363 cluster at $\mathrm{Xq} 26.2$ ), whose classification is due to their identical seed region, which refers to the most important sequence of miRNA for target selection (nucleotides 2-8) (11) (Fig. 1). The deregulation of miR-17 family members is mainly owning to the chromosomal amplifications of miRNA loci on which they reside, for example, 13q31-32 amplification in B-cell lymphoma (12), lung cancer (13) and colorectal adenocarcinoma (14); 7q22 amplification in esophageal adenocarcinoma (15), breast cancer and ovarian cancer (16).

Meanwhile, studies have also found that c-Myc overexpression is correlated with the expression of miR-17-92 cluster in colorectal tumors (14), sporadic Burkitt's lymphoma and diffuse large B-cell lymphoma (17). O'Donnell et al reported that miR-17-5p, miR-92 and miR-106a were upregulated by c-Myc in B-cells, suggesting that c-Myc could regulate the miR-17-92 and miR-106a-363 clusters as well (18). Thus, these findings suggest that, apart from genomic amplification, members of the miR-17 family could also be regulated by c-Myc. Although it is well-known that c-Myc could suppress p21 expression at the transcriptional level, it is still unclear whether c-Myc could regulate p21 at the post- 


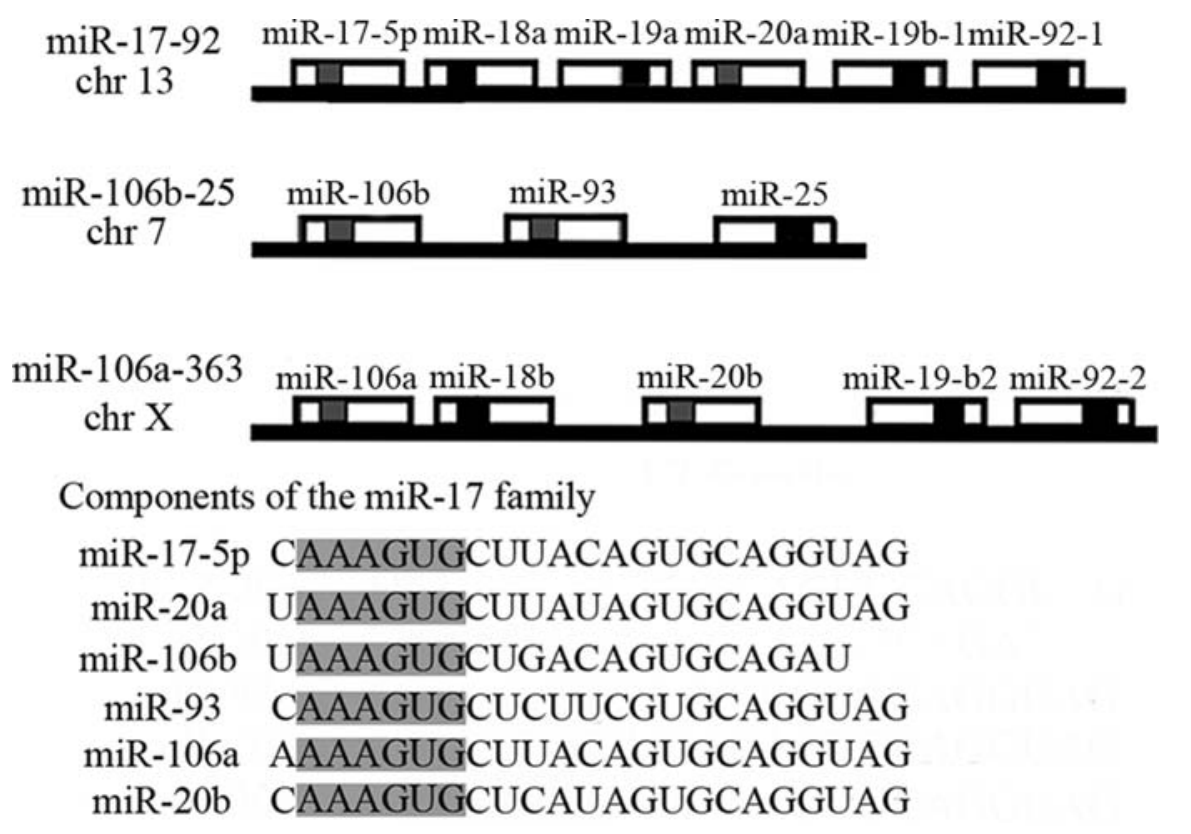

Figure 1. Location and composition of the miR-17 family. Sequences of the miR-17 family members are retrieved from miRBase (release 10.1 ). The chromosomal locations are shown as well, and the identical seed sequences are highlighted in gray.

transcriptional level through members of miR-17 family. To test this issue, we established a stable expression system of c-Myc in HEK293 cells, and found that c-Myc could indeed regulate p21 mRNA at the post-transcriptional level through members of miR-17 family.

\section{Materials and methods}

Plasmid construction and oligonucleotides. To generate the c-Myc expression vector, the open reading frame of the human c-Myc cDNA was cloned into the eukaryotic expression vector pcDNA3 (Invitrogen). The resulting plasmid was designated as pcDNA3/c-Myc. The primers were designed as: forward: 5'CGGGATCCACGATGCCCCTCAACGTTAGC3'; reverse: 5'CGGAATTCTTACGCACAAGAGTTCCGTAG3'.

Antisense oligonucleotides (AS) as well as the universal scrambled oligonucleotides (SC) of miR-17 family members were synthesized as hybrid molecules between deoxyribonucleotides and 2'-O, 4'-C-methylene bridge (locked nucleic acid, LNA) (19) modification of all guanine and cytosine residues (Sangon, Shanghai, China), and their nucleotide sequences were designed as follows: hsa-miR-17-5p AS: 5'ACTACCTGCACTGTAAGCACTTTG3'; hsa-miR-20a AS: 5'CTACCTGCACTATAAGCACTTTA3'; hsa-miR106b AS: 5'ATCTGCACTGTCAGCACTTTA3'; hsa-miR-93 AS: 5'CTACCTGCACGAACAGCACTTTG3'; hsa-miR-106a AS: 5'CTACCTGCACTGTAAGCACTTTT3'; hsa-miR-20b AS: 5'CTACCTGCACTATAAGCACTTTA3'; universal SC: 5'CAGTACTTTTGTGTAGTACAA3'.

Cell culture and transfection. The HEK293 cells were obtained from the American Type Culture Collection (Manassas, VA, USA). HEK293 cells were grown in DMEM (Invitrogen) with $10 \%$ fetal bovine serum (Gibco BRL). These cells were maintained in a humidified $37^{\circ} \mathrm{C}$ incubator with $5 \% \mathrm{CO}_{2}$, fed every 2-3 days with complete medium, and subcultured when confluence was reached. Cells were transfected with Lipofectamine 2000 (Invitrogen) using appropriate plasmid or 100 nM LNA miRNA antisense or scrambled oligonucleotides according to the manufacturer's protocol.

For the establishment of stable transfectants of pcDNA3/ c-Myc, after trasfection, cells were selected with G418 $(800 \mu \mathrm{g} / \mathrm{ml})$, and resistant clones were pooled and cultured. Stable expression of this gene was confirmed by Western blot analysis. The resulting resistant clone population was designated as HEK293/c-Myc, and the control HEK293/ pcDNA3 as well.

RNA extraction and semi-quantitive RT-PCR. Total RNA as well as cytoplasmic and nuclear RNA were extracted from fresh cultured cells with Trizol reagent (Invitrogen, Carlsbad, CA, USA) and Norgen's Cytoplasmic \& Nuclear RNA Purification Kit (Norgen Thorold, ON, Canada) following the manufacturer's protocol, respectively. RNA concentrations were determined with a NanoDrop (NanoDrop Technologies, Inc.).

Two micrograms of extracted RNA was used as template to synthesize cDNA by reverse transcription using M-MLV Reverse Transcriptase (Promega, Madison, WI, USA), in a total volume of $25 \mu \mathrm{l}$ according to the manufacturer's protocol. All PCR reactions were performed using standard PCR conditions: $95^{\circ} \mathrm{C}$ for $5 \mathrm{~min}, 95^{\circ} \mathrm{C}$ for $1 \mathrm{~min}$, annealing $58^{\circ} \mathrm{C}$ for $30 \mathrm{sec}$, extension $72^{\circ} \mathrm{C}$ for $30 \mathrm{sec}$ for 30 cycles, and a final extension at $72^{\circ} \mathrm{C}$ for $10 \mathrm{~min}$. The PCR products were visualized by electrophoresis of $5 \mu 1$ reaction mixture in $2 \%$ agarose gel containing $0.5 \mu \mathrm{g} / \mathrm{ml}$ ethidium bromide, and quantified by densitometry using Gel-Pro Analyzer version 3.1 (Media Cybernetics, Silver Spring, MD, USA). Sequences of the PCR primers for p21 were as follows: forward: 5'GG CTCCTTCCCATCGCTGTCA3'; reverse: 5'GTCACCCTGC 
CCAACCTTAGA3'; ß-actin was used as an internal control, forward: 5'GGCGGCACCACCATGTACCCT3' and reverse: 5'AGGGGCCGGACTCGTCATACT3'.

Conventional real-time PCR and miRNA-specific stem-loop real-time PCR. For miRNA analysis from cultured cells, miRNA was isolated using a mirVana RNA isolation kit (Ambion). Reverse transcription and stem-loop real-time PCR were performed as described (20) by using the TaqMan ${ }^{\circledR}$ MicroRNA Individual Assays, the $\operatorname{TaqMan}^{\circledR}$ MicroRNA Reverse Transcription Kit and the TaqMan ${ }^{\circledR}$ Universal PCR Master Mix without AmpErase ${ }^{\circledR}$ UNG (Applied Biosystems, Foster City, CA, USA). Quantification was carried out by starting with $10 \mathrm{ng}$ of total RNA sample according to the manufacturer's protocol. As a control for normalization, the U6 small nuclear RNA was used. Real-time PCR was performed using an ABI 7300 Sequence Detection System (Applied Biosystems) and fold changes in gene expression were calculated using $2^{-\Delta \Delta C t}$ method (21). The mean miRNA level of the three real-time quantitative PCR experiments was calculated for each case.

The quantification of $\mathrm{p} 21 \mathrm{mRNA}$ and miRNA primary transcripts were carried out by conventional real-time PCR, which was performed in $20 \mu 1$ reaction mixture on an ABI 7300 Sequence Detection System (Applied Biosystems) with Power SYBR Green PCR Master Mix (Applied Biosystems), according to the manufacturer's protocol. $B$-actin was used as an internal control. Detection System and fold changes in gene expression were calculated using $2^{-\Delta \Delta C t}$ method (21). Sequences of the PCR primers were as follows: p21 forward: 5'GGCTCCTTCCCATCGCTGTCA3', reverse: 5'GTCACC CTGCCCAACCTTAGA3'; miR-17-92 forward: 5'CTGTCG CCCAATCAAACTG3', reverse: 5'GTCACAATCCCCACC AAAC3'; miR-106b-25 forward: 5'TGCCCAAGTCTCCGC CTCT3', reverse: 5'CCTTCCTTTCTGTCCTCCC3'; miR106a-363 forward: 5'GAGGTTCATTCACATTCAGTAACA ATCTG3', reverse: 5'GTTGGCGACATTCCTGATCAGC3'; ß-actin forward: 5'GGCGGCACCACCATGTACCCT3', reverse: 5'AGGGGCCGGACTCGTCATACT3'.

Western blot analysis. Cells were transfected with respective oligonucleotides and were harvested after $24 \mathrm{~h}$ and lysed in RIPA buffer (Sigma), then Western blot analysis was performed with the use of conventional protocols as described previously (22). Total proteins were separated by SDS-PAGE, and then transferred onto nitrocellulose membranes. The antibodies and dilutions used including anti-ß-actin (AC-15; 1:5000; Sigma-Aldrich), anti-c-Myc (551101; 1:1000 BD Pharmingen $^{\mathrm{TM}}$ ), anti-p21 ${ }^{\text {Wafl/Cip1 }}$ (2946; 1:1000 Cell Signaling Technology). After extensive washing, the membranes were incubated with HRP (horseradish peroxidase) conjugated secondary antibody (Zhongshan Goldenbridge Biotechnology Co., Beijing, China) for $1 \mathrm{~h}$ at room temperature and developed using Luminol chemiluminescence detection kit (Santa Cruz). Membranes were reprobed for $\beta$-actin to normalize for loading and to allow for accurate quantification. Protein expression was quantified using a Gel EDAS 293 analysis system (Cold Spring USA Corp., Cherry Hill, NJ, USA) and Gel-Pro Analyzer 3.1 software (Media Cybernetics, Bethesda, MD, USA).
A
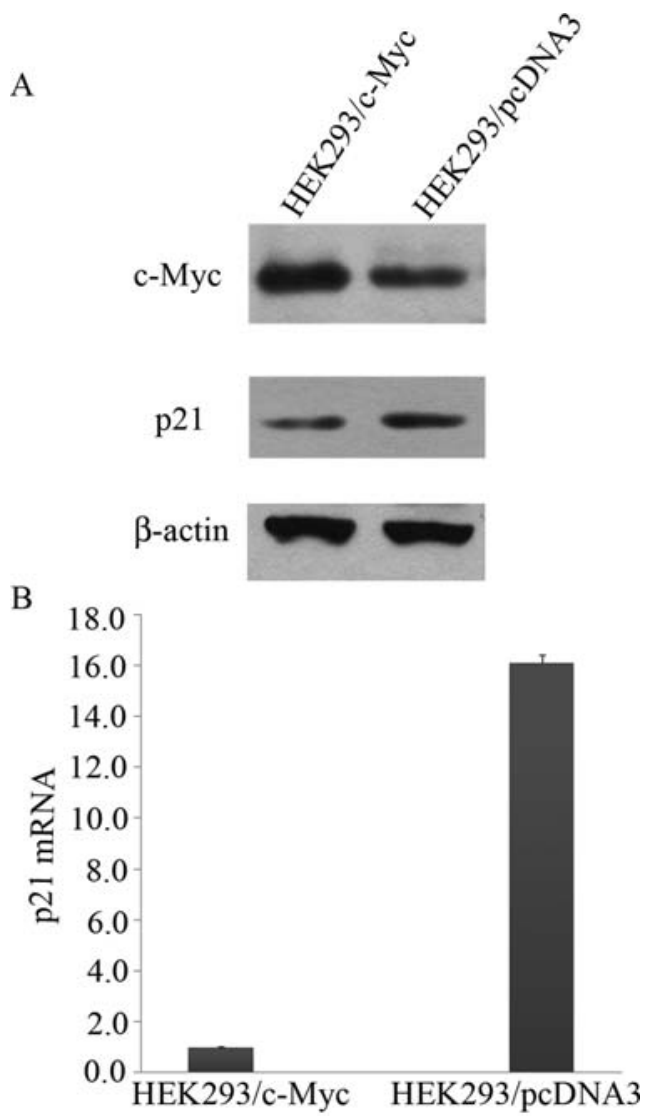

Figure 2. Stable overexpression of c-Myc in HEK293 cells suppress the expression of p21. (A) Exponentially proliferating HEK293 cells were transfected with pcDNA3/c-Myc or pcDNA3 and selected with G418 to obtain stable transfectants. G418-resistant clones were pooled and clones were cultured and protein lysates were prepared by SDS-PAGE and immunoblotted with antibodies to $\mathrm{c}-\mathrm{Myc}$ or $\mathrm{p} 21$; expression levels were normalized using $\beta$-actin as an internal control. (B) p21 mRNA levels were examined by real-time PCR. Human $\beta$-actin gene was used as an internal control, and p21 mRNA of HEK293/c-Myc was designated as unit 1. Means $\pm \mathrm{SD}(\mathrm{n}=3) ; \mathrm{p}<0.05$.

Statistical analysis. The results were expressed as the means \pm SD. A Student's two-tailed non-paired t-test was used to determine the statistical significance. $\mathrm{p}<0.05$ was considered statistically significant.

\section{Results}

Expression of p21 mRNA in the nucleus and cytoplasm of c-Myc stable transfectants and its control. HEK293 cells were transfected with pcDNA3/c-Myc or pcDNA3 and selected with G418 to obtain stable transfectants. The resulting pool of stable transfectants was designated as HEK293/c-Myc and HEK293/pcDNA3. Western blotting and detection of p21 mRNA by real-time PCR were performed (Fig. 2), which demonstrated that the expression of p21 was fundamentally depressed by stable overexpression of c-Myc, at both transcriptional and expressional level.

As is known, compared with the mRNA level in the nucleus which is the result of transcriptional regulation, the mRNA level in the cytoplasm are the collective results of both transcriptional regulation and post-transcriptional regulation. After extraction and purification of nuclear and 

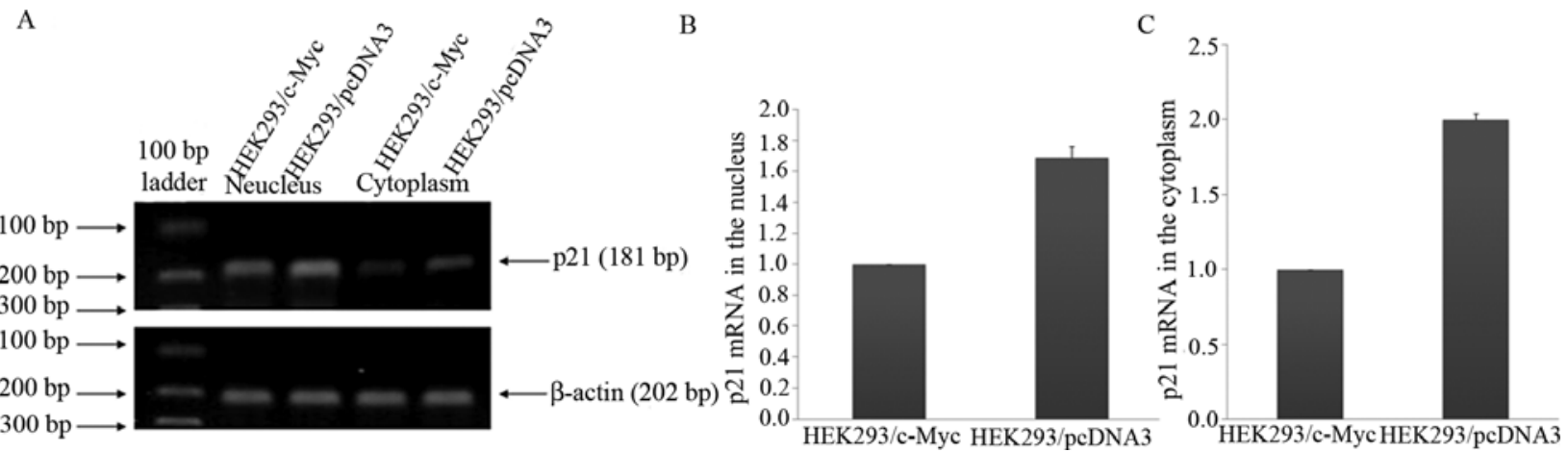

Figure 3. Expression of p21 mRNA in the nucleus and cytoplasm of HEK293/c-Myc and HEK293/pcDNA3 transfectants. (A) A p21 cDNA fragment of 181-bp in size and a 3 -actin cDNA fragment of 202-bp were detected by semi-quantitive RT-PCR. (B) p21 mRNA in the nucleus and cytoplasm (C) were both analyzed by real-time PCR. Human $\beta$-actin gene was used as an internal control, and p21 mRNA in the nucleus or the cytoplasm of HEK293/c-Myc was designated as unit 1 , respectively. Means $\pm \mathrm{SD}(\mathrm{n}=3) ; \mathrm{p}<0.05$.

cytoplasmic RNA of HEK293/c-Myc and HEK293/pcDNA3 transfectants, semi-quantitive RT-PCR was performed (Fig. 3A). Furthermore, quantified expression differences of p21 mRNA in these components were detected by real-time PCR. The expression of p21 mRNA in the nucleus of HEK293/ pcDNA3 was 1.7 times higher than its counterpart of HEK293/c-Myc (p<0.05; Fig. 3B), as well as the expression in the cytoplasm, which displayed 2.0 times higher in HEK293/pcDNA3 than in HEK293/c-Myc (p<0.05, Fig. 3C). While the mRNA level was $>20$-times higher in the nucleus than in the cytoplasm either in HEK293/c-Myc or HEK293/ pcDNA3 (data not shown).

Regulation of pri-miRNA transcripts and mature miRNAs in miR-17 family by overexpression of c-Myc. miR-17-92 cluster is the pri-miRNA of miR-17-5p and miR-20a, while its two paralogs miR-106b-25 and miR-106a-363, are the pri-miRNAs of miR-106b and miR-93, miR-106a and miR20b, respectively. Since miRNAs are transcribed by RNA polymerase II as long primary transcripts (pri-miRNAs) that undergo sequential processing to produce mature miRNAs, primers for real-time PCR was designed to detect the expression of pri-miRNA transcripts in HEK293/c-Myc and HEK293/pcDNA3 transfectants (Fig. 4A). The expression level of miR-17-92 showed 5.1-fold increase in HEK293/ c-Myc compared with its expression in HEK293/pcDNA3 $(\mathrm{p}<0.05)$, as well as for miR-106a-363, which displayed 3.8 -fold increase $(\mathrm{p}<0.05)$, whereas the expression of miR106b-25 exhibited no obvious difference $(\mathrm{p}<0.05)$.

Furthermore, we applied stem-loop real-time PCR to evaluate expression alteration of each miRNA member in miR-17 family. Fig. 4B showed 2.0-fold $(\mathrm{p}<0.05)$ increase of miR-17-5p in HEK293/c-Myc compared with its expression in HEK293/pcDNA3, for miR-20a, which diaplayed 2.4-fold increase $(\mathrm{p}<0.05)$, and for miR-106a, 1.9-fold increase $(p<0.05)$. While the expression of miR-106b and miR-93 was nearly the same in both HEK293/c-Myc and HEK293/ pcDNA3, and the expression of miR-20b in both transfectants was very low.

Antagonizing the expression of miR-17 family members by antisense oligonucleotide transfection restores p 21 expression

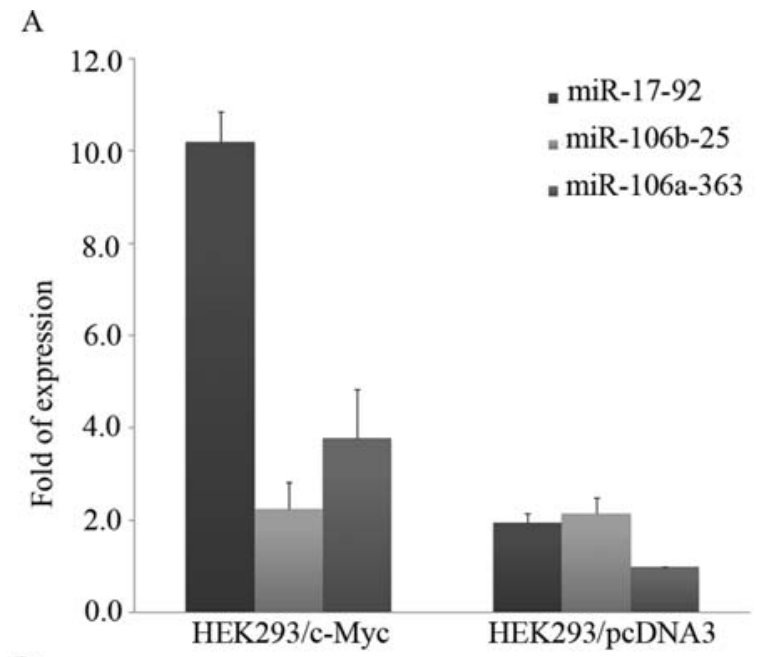

B

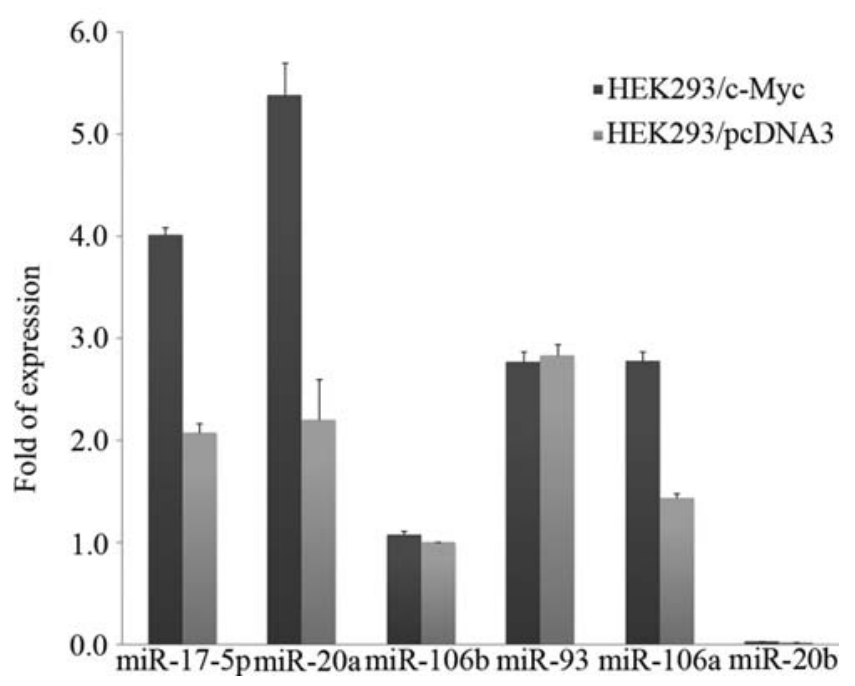

Figure 4. Expression of pri-miRNA transcripts and mature miRNAs of miR-17 family in HEK293/c-Myc and HEK293/pcDNA3 transfectants. (A) Expression of miR-17-92, miR-106b-25 and miR-106a-363 mRNA was measured by real-time PCR. Human B-actin gene was used as an internal control, and miR-106a-363 mRNA of HEK293/pcDNA3 was designated as unit 1. (B) Expression of mature microRNAs in miR-17 family was analyzed by TaqMan miRNA assays using stem-loop real-time PCR. U6 small nuclear RNA was used as an internal control, miR-106b of HEK293/ pcDNA3 was designated as unit 1 . Means \pm SD $(n=3) ; p<0.05$. 
A

Site A: position 468-474 of human p21/CDKN1A 3'-UTR

Site A of p21 5'-AGAAGUAAACAGAUGGCACUUUG-3' hsa-miR-17-5p 3'-GAUGGACGUGACAUUCGUGAAAC-5'

Site A of p21 5'-AGAAGUAAACAGAUGGCACUUUG-3' |||||| $\mid$

hsa-miR-20a 3'-GAUGGACGUGAUAUUCGUGAAAU-5'

Site A of p21 5'-AGAAGUAAACAGAUGGCACUUUG-3' hsa-miR-106b 3'-UAGACGUGACAGUCGUGAAAU-5'

Site A of p21 5'-AGAAGUAAACAGAUGGCACUUUG-3' hsa-miR-93 3'-GAUGGACGUGCUUGUCGUGAAAC-5'

Site A of p21 5'-AGAAGUAAACAGAUGGCACUUUG-3' hsa-miR-106a 3'-GAUGGACGUGACAUUCGUGAAAA-5'

Site A of p21 5'-AGAAGUAAACAGAUGGCACUUUG-3' hsa-miR-20b 3'-GAUGGACGUGAUACUCGUGAAAC-5'

B

Site B: position 1148-1154 of human p21/CDKN1A 3'-UTR Site B of p21 5'-CCUCCCCAGUUCAUUGCACUUUG-3'

hsa-miR-17-5p 3'-GAUGGACGUGACAUUCGUGAAAC-5'

Site B of p21 5'-CCUCCCCAGUUCAUUGCACUUUG-3' hsa-miR-20a 3'-GAUGGACGUGAUAUUCGUGAAAU-5'

Site B of p21 5'-CCUCCCCAGUUCAUUGCACUUUG-3' hsa-miR-106b 3'-UAGACGUGACAGUCGUGAAAU-5,

Site B of p21 5'-CCUCCCCAGUUCAUUGCACUUUG-3' hsa-miR-93 3'-GAUGGACGUGCUUGUCGUGAAAC-5'

Site B of p21 5'-CCUCCCCAGUUCAUUGCACUUUG-3'

hsa-miR-106a 3'-GAUGGACGUGACAUUCGUGAAAA-5'

Site B of p21 5'-CCUCCCCAGUUCAUUGCACUUUG-3' hsa-miR-20b 3'-GAUGGACGUGAUACUCGUGAAAC-5',

Figure 5.p21 is a common target of the miR-17 family members.

much more strongly in the presence of $c-M y c$. miRNAs in the miR-17 family are identical in their seed sequences and are similar in their 3'-half (Fig. 1), which suggests that they may have similar functions. Interestingly, all the members of miR-17 family are predicted to control p21, according to the results of three different well-known target prediction programs, TargetScan, PicTar, and miRBase (Fig. 5).

To determine whether c-Myc could repress p21 expression through up-regulated miR-17 family members at the posttranscriptional level, we transfected antisense oligonucleotides (AS) and their control (scramble oligonucleotides, SC) of miR-17 family into HEK293/c-Myc and HEK293/pcDNA3 to suppress the endogenous expression of mature miRNAs, respectively. AS used in these assays were complementary to their corresponding mature miRNA and both AS and SC were modified by LNA at each $\mathrm{G}$ and $\mathrm{C}$ base. The inhibitory effects were examined by stem-loop real-time PCR (data not shown). Since the universal SC sequence was from miRNA of C. elegans without any homology to human miRNAs, transfection of it had nearly no influence on the expression of other human miRNAs. Compared with SC, each specific AS could significantly reduce the endogenous expression of corresponding mature miRNAs of miR-17 family, respectively. Western blotting and real-time PCR were carried out to evaluate p21 expression (Fig. 6).

As Fig. 6A, C and $\mathrm{E}$ show, the degree of $\mathrm{p} 21$ restoration achieved by inhibiting endogenous mir-17-5p, miR-20a and miR-106a expression was much stronger in HEK293/c-Myc than in HEK293/pcDNA3, especially the result from inhibition of miR-17-5p. While there was no obvious up-regulation of p21 induced by miR-93 inhibition either in HEK293/c-Myc or in HEK293/pcDNA3 (Fig. 6G). The result of miR-106b inhibition was similar to miR-93 (data not shown).

We also tested the effect of miRNA inhibition of p21 mRNA level by real-time PCR. The p 21 mRNA was elevated by miR-17-5p AS and miR-106a AS treatment compared with SC in both HEK293/c-Myc and HEK293/pcDNA3 (Fig. 6B and F). Thus, p21 was likely to be suppressed by miR-17-5p and miR-106a through both mRNA decay and translational inhibition. While, the p21 mRNA was only slightly increased by miR-20a, miR-93 and miR-106b (similar result to miR-93, data not shown) AS treatment (Fig. 6D and H), indicating their repression of p21 may be mainly due to translational inhibition.

\section{Discussion}

The nuclear phosphoprotein c-Myc is a central regulator of cellular proliferation and cell growth. Deregulated expression of c-Myc has been demonstrated in many types of cancer $(23,24)$. Being a multifaceted protein, c-Myc not only activates growth-promoting genes, but also tends to down-regulate several growth arrest genes including gas1, gadds, p15, p27, and p21 (23). As a potent regulator of p21, it has been found that c-Myc may employ a multitude of pathways to repress p21 at the transcriptional level $(3,6,23,25)$.

In the present study, we demonstrate that c-Myc-mediated repression of p21 not only takes place at the transcriptional level, but also exists at the post-transcriptional level through up-regulation of some members of miR-17 family (miR-175p, miR-20a, and miR-106a).

The detection of p21 mRNA in the nucleus of HEK293/ c-Myc shows 0.7-fold decrease compared with that in HEK293/pcDNA3, while p21 mRNA in the cytoplasm shows 1-fold decrease in HEK293/c-Myc compared to its control. The increased reduction of p21 mRNA in the cytoplasm demonstrates that, under the circumstances of c-Myc stable overexpression, some other regulatory mechanism must exist in the cytoplasm (namely, at the post-transcriptional level), besides the effect of transcriptional suppression of $\mathrm{p} 21$ mRNA in the nucleus. Meanwhile, much more excessive expression of p21 mRNA in the nucleus than in the cytoplasm indicates that, only a small amount of mRNA is transported to the cytoplasm from the nucleus and takes part in protein biosynthesis.

Overexpression of c-Myc elevates the expression of miR-17-5p, miR-20a, and miR-106a in miR-17 family, in 

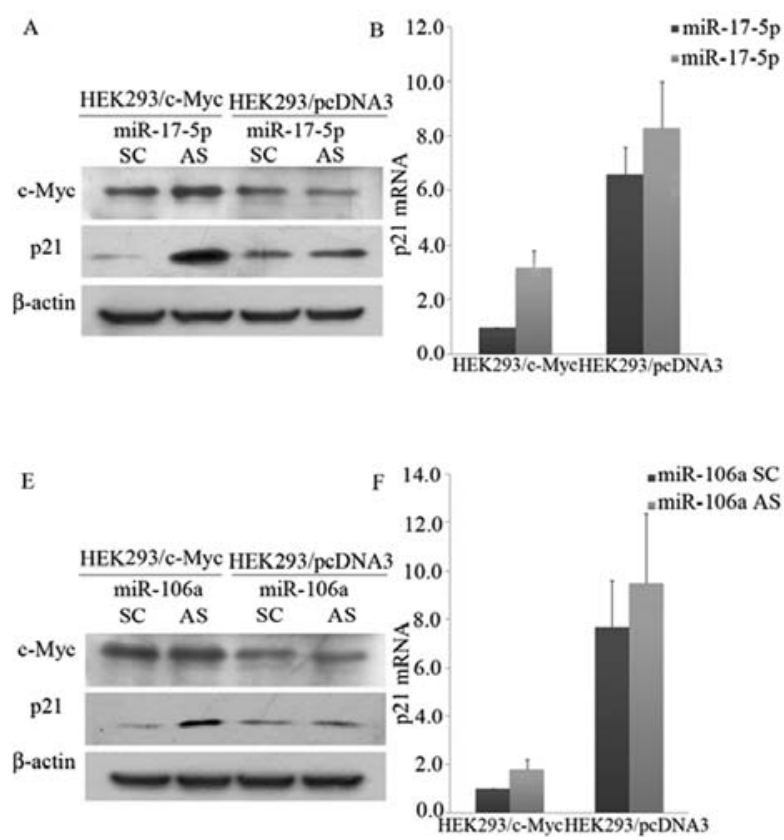
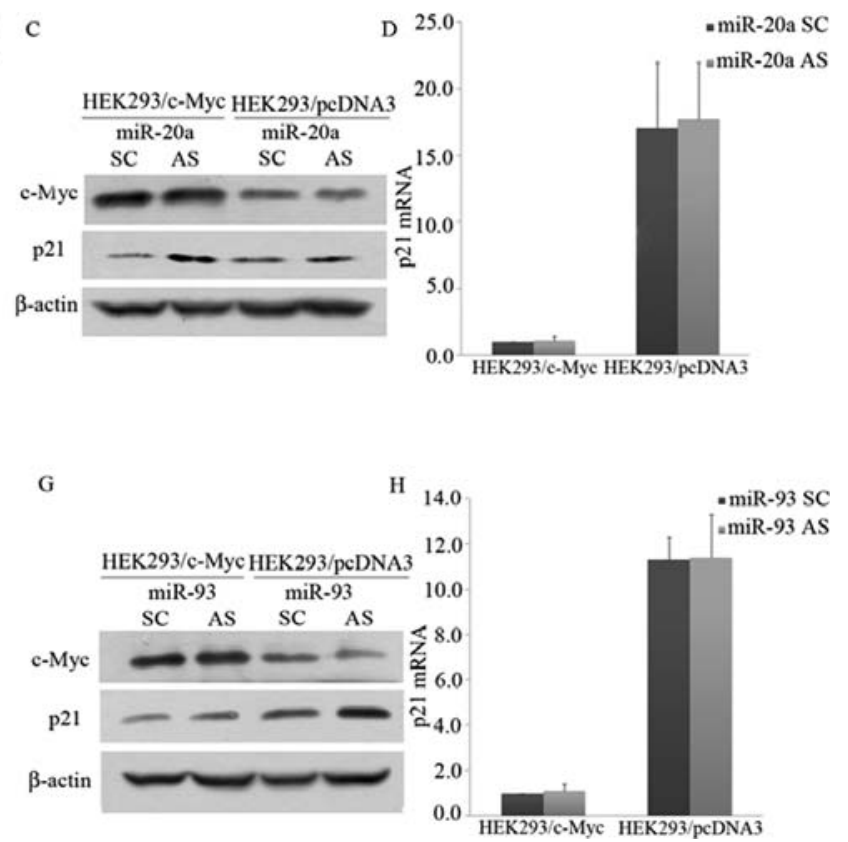

Figure 6. Inhibition of miR-17 family member expression respectively by antisense oligonucleotide transfection in HEK293/c-Myc and HEK293/pcDNA3 transfectants led to different restoration of p21 mRNA and protein. Western blotting (A, C, E and G) of HEK293/c-Myc and HEK293/pcDNA3 transfectants which were transiently transfected with LNA-labeled miRNA (miR-17-5p, miR-20a, miR-106a, miR-93) antisense and scramble oligonucleotides (100 nM) for $24 \mathrm{~h}$. The protein lysates were prepared and separated by SDS-PAGE and immunoblotted with antibodies to c-Myc or p21; expression levels were normalized using $\beta$-actin as an internal control. p21 mRNA was also detected respectively (B, D, F and H) by real-time PCR. Human ß-actin gene was used as an internal control and mRNA of HEK293/c-Myc treated with scramble oligonucleotides was designated as unit 1 . Means \pm SD ( $\mathrm{n}=3$ ); $\mathrm{p}<0.05$.

addition, increases the expression of their primary transcripts (miR-17-92 and miR-106a-363 cluster), as reported by O'Donnell et al (18).

The slight elevation of p21 protein level in HEK293/ pcDNA3 shown in Fig. 6A, C, E and G, achieved by inhibiting endogeneous expression of each member in miR17 family through antisense oligonucleotides transfection, indicates that p21 is a target of miR-17 family. While the prominent increase of p21 protein by the same treatment in HEK293/c-Myc demonstrates that the effect of miR-17-5p, miR-20a, and miR-106a on p21 is much stronger in the presence of c-Myc. Namely, the suppression effect of p21 by $\mathrm{c}-\mathrm{Myc}$ is partly due to the elevated expression of miR-17-5p, miR-20a, and miR-106a at the post-transcriptional level. For miR-106b and miR-93, it seems that they could not be regulated by c-Myc (Fig. 4), and antagonizing their expression by $\mathrm{AS}$ and $\mathrm{SC}$ transfection has no difference between HEK293/c-Myc and HEK293/pcDNA3 (Fig. 6G and H).

Regulations of genes are complicated and sophisticated. Besides p21, E2F1 and Bim are also targets of miR-17 family $(18,26-28)$, and at the same time, transactivated by c-Myc at the transcriptional level (29-31). Take E2F1 as an example, as a master regulator of cell cycle that promotes the G1/S transition, E2F1 is induced by c-Myc potently (32). While the c-Myc-mediated induction of E2F1 translation is limited by $\mathrm{miR}-17-5 \mathrm{p}$ and miR-20a at the post-transcriptional level (18), providing a mechanism to dampen reciprocal activation of c-myc to E2F1.

From this and previous studies, we know that c-Myc controls its target genes through a very precise regulation. It could either subtly regulate gene expression by activating their transcription and limiting their translation simultaneously, or repress gene expression at both transcriptional and posttranscriptional level. However, it is still unclear, whether any other regulatory pathway exists at the post-transcriptional level which is controlled by c-Myc, if it does, how do they collaborate with each other, and what is the biological function? Further investigation is still needed to elucidate these issues.

\section{Acknowledgements}

This work was supported by National Natural Science Foundation (39925020, 30721001), National Basic Research Program (2004CB518701) and State Key Laboratory of Molecular Oncology program (SKL-2007-03), P.R. China.

\section{References}

1. Santamaria D and Ortega S: Cyclins and CDKS in development and cancer: lessons from genetically modified mice. Front Biosci 11: 1164-1188, 2006.

2. Claassen GF and Hann SR: A role for transcriptional repression of $\mathrm{p} 21^{\mathrm{CIPl}}$ by c-Myc in overcoming transforming growth factor beta-induced cell-cycle arrest. Proc Natl Acad Sci USA 97: 9498-9503, 2000.

3. Gartel AL, Ye X, Goufman E, Shianov P, Hay N, Najmabadi F and Tyner AL: Myc represses the p21(WAF1/CIP1) promoter and interacts with Sp1/Sp3. Proc Natl Acad Sci USA 98: 4510-4515, 2001.

4. Mitchell KO and El-Deiry WS: Overexpression of c-Myc inhibits $\mathrm{p} 21^{\mathrm{WAF} 1 / \mathrm{CIP} 1}$ expression and induces S-phase entry in 12-Otetradecanoylphorbol-13-acetate (TPA)-sensitive human cancer cells. Cell Growth Differ 10: 223-230, 1999.

5. Seoane J, Le HV and Massague J: Myc suppression of the p21(Cip1) Cdk inhibitor influences the outcome of the p53 response to DNA damage. Nature 419: 729-734, 2002. 
6. Wu S, Cetinkaya C, Munoz-Alonso MJ, et al: Myc represses differentiation-induced p $21^{\mathrm{CIP} 1}$ expression via Miz-1-dependent interaction with the p21 core promoter. Oncogene 22: 351-360, 2003.

7. Van de Wetering M, Sancho E, Verweij C, et al: The betacatenin/TCF-4 complex imposes a crypt progenitor phenotype on colorectal cancer cells. Cell 111: 241-250, 2002.

8. Ivanovska I, Ball AS, Diaz RL, et al: MicroRNAs in the miR$106 \mathrm{~b}$ family regulate $\mathrm{p} 21 / \mathrm{CDKN} 1 \mathrm{~A}$ and promote cell cycle progression. Mol Cell Biol 28: 2167-2174, 2008.

9. Petrocca F, Visone R, Onelli MR, et al: E2F1-regulated microRNAs impair TGFbeta-dependent cell-cycle arrest and apoptosis in gastric cancer. Cancer Cell 13: 272-286, 2008.

10. Kim YK, Yu J, Han TS, et al: Functional links between clustered microRNAs: suppression of cell-cycle inhibitors by microRNA clusters in gastric cancer. Nucleic Acids Res 37: 1672-1681, 2009.

11. Mendell JT: miRiad roles for the miR-17-92 cluster in development and disease. Cell 133: 217-222, 2008.

12. Ota A. Tagawa H, Karnan S, Tsuzuki S, Karpas A, Kira S Yoshida $\mathrm{Y}$ and Seto M: Identification and characterization of a novel gene, $\mathrm{C} 13$ orf 25, as a target for 13q31-q32 amplification in malignant lymphoma. Cancer Res 64: 3087-3095, 2004.

13. Hayashita Y, Osada H, Tatematsu Y, et al: A polycistronic microRNA cluster, miR-17-92, is overexpressed in human lung cancers and enhances cell proliferation. Cancer Res 65: 9628-9632, 2005.

14. Diosdado B, van de Wiel MA, Terhaar Sive Droste JS, et al: MiR-17-92 cluster is associated with 13q gain and c-myc expression during colorectal adenoma to adenocarcinoma progression. Br J Cancer 101: 707-714, 2009.

15. Kan $\mathrm{T}$, Sato $\mathrm{F}$, Ito $\mathrm{T}$, et al: The miR-106b-25 polycistron, activated by genomic amplification, functions as an oncogene by suppressing p21 and Bim. Gastroenterology 136: 1689-1700, 2009.

16. Zhang L, Huang J, Yang N, et al: microRNAs exhibit high frequency genomic alterations in human cancer. Proc Natl Acad Sci USA 103: 9136-9141, 2006.

17. Tagawa H, Karube K, Tsuzuki S, Ohshima K and Seto M: Synergistic action of the microRNA-17 polycistron and Myc in aggressive cancer development. Cancer Sci 98: 1482-1490, 2007.

18. O'Donnell KA, Wentzel EA, Zeller KI, Dang CV and Mendell JT: c-Myc-regulated microRNAs modulate E2F1 expression. Nature 435: 839-843, 2005.
19. Gleave ME and Monia BP: Antisense therapy for cancer. Nat Rev Cancer 5: 468-479, 2005.

20. Chen C, Ridzon DA, Broomer AJ, et al: Real-time quantification of microRNAs by stem-loop RT-PCR. Nucleic Acids Res 33: e179, 2005

21. Livak KJ and Schmittgen TD: Analysis of relative gene expression data using real-time quantitative PCR and the 2(-Delta Delta C(T)) method. Methods 25: 402-408, 2001.

22. Zhou C, Liu S, Zhou X, et al: Overexpression of human pituitary tumor transforming gene (hPTTG), is regulated by beta-catenin/ TCF pathway in human esophageal squamous cell carcinoma. Int J Cancer 113: 891-898, 2005.

23. Gartel AL and Shchors K: Mechanisms of c-myc-mediated transcriptional repression of growth arrest genes. Exp Cell Res 283: 17-21, 2003.

24. Nesbit CE, Tersak JM and Prochownik EV: MYC oncogenes and human neoplastic disease. Oncogene 18: 3004-3016, 1999.

25. Brenner C, Deplus R, Didelot C, et al: Myc represses transcription through recruitment of DNA methyltransferase corepressor. EMBO J 24: 336-346, 2005.

26. Fontana L, Fiori ME, Albini S, et al: Antagomir-17-5p abolishes the growth of therapy-resistant neuroblastoma through p21 and BIM. PLoS One 3: e2236, 2008.

27. Hossain A, Kuo MT and Saunders GF: Mir-17-5p regulates breast cancer cell proliferation by inhibiting translation of AIB 1 mRNA. Mol Cell Biol 26: 8191-8201, 2006.

28. Scherr M, Venturini L, Battmer K, Schaller-Schoenitz M, Schaefer D, Dallmann I, Ganser A and Eder M: Lentivirusmediated antagomir expression for specific inhibition of miRNA function. Nucleic Acids Res 35: e149, 2007.

29. Dang CV, O'Donnell KA and Juopperi T: The great MYC escape in tumorigenesis. Cancer Cell 8: 177-178, 2005.

30. Leone G, De Gregori J, Sears R, Jakoi L and Nevins JR: Myc and Ras collaborate in inducing accumulation of active cyclin E/Cdk2 and E2F. Nature 387: 422-426, 1997.

31. Fernandez PC, Frank SR, Wang L, Schroeder M, Liu S, Greene J, Cocito A and Amati B: Genomic targets of the human c-Myc protein. Genes Dev 17: 1115-1129, 2003.

32. Matsumura I, Tanaka H and Kanakura Y: E2F1 and c-Myc in cell growth and death. Cell Cycle 2: 333-338, 2003. 